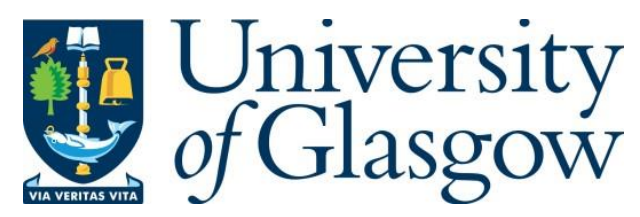

Lim, L. H. I. and Loh, A. P. (2006) Identification of Frequency Ranges for Subharmonic Oscillations in a Relay Feedback System. In: American Control Conference 2006, Minneapolis, MN, USA, 14-16 Jun 2006, pp. 3789-3794. ISBN 9781424402090.

There may be differences between this version and the published version. You are advised to consult the publisher's version if you wish to cite from it.

http://eprints.gla.ac.uk/167103/

Deposited on: 17 August 2018

Enlighten - Research publications by members of the University of Glasgow http://eprints.gla.ac.uk 


\title{
Identification of Frequency Ranges for Subharmonic Oscillations in a Relay Feedback System
}

\author{
Lim L H* $\mathrm{H}^{*}$ Loh A $\mathrm{P}^{\dagger}$ \\ *Email: g0402566@nus.edu.sg \\ ${ }^{\dagger}$ Dept of Electrical \& Computer Engineering \\ National University of Singapore \\ Email : elelohap@nus.edu.sg
}

\begin{abstract}
This paper examines the behaviour of a single loop relay feedback system (RFS) which is driven by an external signal. It is well known that such a RFS exhibits a variety of oscillation patterns including forced and subharmonic oscillations (SO). This paper focuses on the conditions for SO. It will be shown that for an external signal with a fixed amplitude, it is possible for SO with different orders to occur simply by changing the frequency of the external signal. Similarly, for an external signal with a fixed frequency, it is possible for SO with different orders to occur when the amplitude of the external signal is varied. The conditions under which these different scenarios will occur are explored. An analysis of these conditions identifies the frequency ranges where certain orders of SO are possible for a given amplitude of the external signal. The effects of the initial conditions on the SO are illustrated and discussed. Simulation studies are presented to illustrate the results.
\end{abstract}

Keywords: relay feedback systems, subharmonic oscillations

\section{INTRODUCTION}

Relay feedback as a control technique has received great attention since 1887 when Hawkins discovered that a temperature control system has a tendency to oscillate under discontinuous control. Continued attention on relay feedback was due to its widespread use in mechanical and electromechanical applications. Since then, the study of the relay feedback system (RFS) has been spurred on by the modern developments in supervisory switched systems and variable structure controllers. The latest application of the relay feedback in the area of modern control is the use of its limit cycling properties in controller tuning and identification [1][3].

The application of the RFS in a wide range of settings has prompted extensive studies on its behaviour. Due to the switching nature of the relay, the RFS is essentially nonlinear as the output of the relay is discontinuous at its switching instants. The complex dynamics exhibited by the relay resulted in various interesting phenomena such as the existence of fast switches, sliding motion and limit cycling. The existence of fast switches and sliding motion has been extensively studied in [4], [1] and [5] while the global stability of limit cycles in the RFS was proven in [6]. In that paper, the quadratic stability of associated Poincare maps was checked by solving linear matrix inequalities using computational tools.

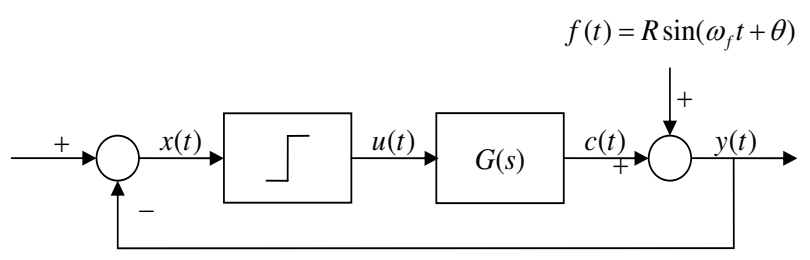

Fig. 1. Single loop with external forcing signal.

While all these studies were going on, little attention was given to externally forced RFS even though the phenomenon of forced oscillations (FO) have been known since the days of the Huygens' clock. More recently, with the extension of the auto-tuning techniques to multivariable systems, a better understanding of FO was given in [7]. This paper is an extension of the results in [7], with the focus being on another common behaviour, namely that of subharmonic oscillations (SO). When the external signal, $f(t)$, of frequency $\omega_{f}$ induces oscillations of frequency $\omega_{f}$ in the RFS, then FO is said to have taken place. When an $f(t)$ of frequency $\nu \omega_{f}$ induces oscillations of frequency $\omega_{f}$, then $\mathrm{SO}$ of order $\nu$ is said to have taken place. As the ideal relay is symmetrical about the origin, only odd subharmonics are possible. Compared to FO, SO behaviours are even more rarely explored in much of the current RFS literature.

There are many methods used to predict the existence of oscillations in relay feedback systems. The most common approach, the describing function approach is an approximate method used to predict the existence of oscillations. Other methods by Tsypkin [2] and Atherton [8] attempted to identify the amplitude of the external forcing signal required for FO and SO. The studies in [2], [8] and [7] on the minimum amplitude of $f(t)$ required for $\mathrm{FO}$ and $\mathrm{SO}$ did not reveal the full extent of the problem on the existence of SO. The prediction of SO is more difficult than FO because they cannot be observed for all $f(t)$ of arbitrary frequencies. Furthermore, under different conditions, the order of SO also changes. This problem is illustrated in Fig. 2 where the order of SO changes from $\nu=7$ to $\nu=9$ when $\theta$ changes from $3.7726 \mathrm{rad}$ to $0 \mathrm{rad}$ with all other conditions unchanged. The problem of predicting which order of $\mathrm{SO}$ is possible is further complicated when the initial value of the state vector, $z_{0}$, the amplitude, $R$, and frequency, $\omega_{f}$ of $f(t)$ are considered.

This paper examines the conditions for SO in the RFS of Fig. 1. Specifically, the conditions under which different 
orders of SO can occur are explored. The analysis naturally leads to an interesting variety of possibilities, including the range of frequencies of $f(t)$ where certain orders of SO are possible.

The paper is organised as follows. The problem formulation is presented in Section II and the necessary and sufficient conditions for periodic switching and their analysis are shown in Section III. Section IV analyses the existence of the SO orders, $\nu$, and presents the simulation results. Conclusions are given in Section V.

\section{PROBlem Formulation}

Consider the RFS with an external forcing signal, $f(t)$, as shown in Fig. 1. The linear system, $G(s)$, is assumed to have a state space description given by

$$
\begin{aligned}
& \dot{z}(t)=A z(t)+B u(t-L) \\
& c(t)=C z(t) \\
& u(t)=\left\{\begin{array}{cc}
h & y(t)<0 \\
-h & y(t) \geq 0
\end{array}\right.
\end{aligned}
$$

where $h>0, u, c \in R$ are the input and output, respectively, $z \in R^{m \times 1}$ is the state vector, $L>0$ is the time delay between $u$ and $c, A \in R^{m \times m}$ is Hurwitz and assumed to be non-singular, $B \in R^{m \times 1}$ and $C \in R^{1 \times m}$. In the frequency domain,

$$
G(s)=e^{-s L} C(s I-A)^{-1} B
$$

and satisfies $\lim _{s \rightarrow \infty} G(s)=0$.

In the externally forced RFS,

$$
y(t)=c(t)+f(t)
$$

where $f(t)=R \sin \left(\omega_{f} t+\theta\right)$ is the external forcing signal.

The problem we address is that of subharmonic oscillations. From our previous work in [7], it was shown that FO can always occur (particularly for delayed system) when the amplitude of $f(t)$ is sufficiently large. However, this is not true in the case of SO. The occurrence of SO of specific order is very much dependent on the initial conditions, the amplitude, $R$, the frequency, $\omega_{f}$, and phase, $\theta$ of the external signal. This phenomenon is further complicated by the existence of SO of different orders, simply by varying any one of the factors above. The search for SO is also elusive

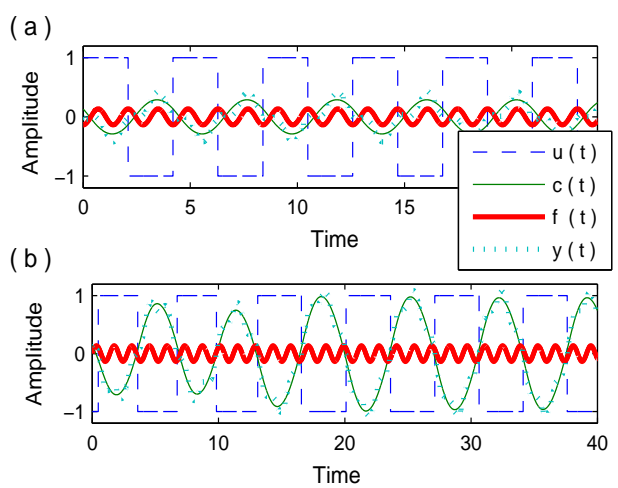

Fig. 2. (a) SO of $\nu=7$ obtained with $\theta=3.7726$. (b) SO of $\nu=9$ obtained with $\theta=0$. because SO does not necessarily occur for arbitrary $f(t)$. Thus the prediction of the frequency ranges of $f(t)$ where $\mathrm{SO}$ is possible becomes useful and important.

Our analysis starts with the necessary and sufficient conditions required for periodic oscillations to occur. Specifically, we determine the minimum amplitude, $R$, of $f(t)$ where SO of a certain order can occur in the RFS with different $G(s)$. Secondly, we derive a procedure that predicts the frequency ranges of $f(t)$ for which SO of certain orders can take place. It will also be shown how the order of the SO is affected by initial conditions in the system, including the initial state vector of $G(s)$ and the phase offset, $\theta$ of $f(t)$. A variety of interesting behaviours will be shown as a result of the analysis.

\section{NECESSARY AND SUFFICIENT CONDITIONS FOR PERIODIC SWITCHING}

When symmetric unimodal limit cycles of any frequency $\omega$ occur, the relay switches at exactly two instants per period. For steady state analysis, it is common practice to re-define the time scale. Unless otherwise stated, $t$ denotes the time taken with respect to any positive switching edge of the relay output during periodic switching. Necessary conditions [8] can therefore be derived as follows. At the switching instants, $t=0$ and $t=\pi / \omega^{-}$, the output, $y(t)$, of the RFS and the input, $x(t)$ of the relay satisfies

$$
\begin{aligned}
& x(0)=-y(0)=0, x\left(\frac{\pi}{\omega}^{-}\right)=-y\left(\frac{\pi}{\omega}^{-}\right)=0 \\
& \dot{x}(0)=-\dot{y}(0)>0, \dot{x}\left(\frac{\pi}{\omega}^{-}\right)=-\dot{y}\left(\frac{\pi}{\omega}^{-}\right)<0
\end{aligned}
$$

where $t=0$ corresponds to the positive switching edge of the relay at steady state. Conditions (4) and (5) examine the input signal to the relay at only the switch points corresponding to every half period of the oscillation frequency, thereby ensuring the correct switching for steady state periodic oscillations to take place. Since these conditions do not examine the relay input signals in between every switching instant, they are not sufficient to guarantee switchings at only these points. It is possible that additional switchings may take place in between any half period, thereby destroying the periodicity of the relay outputs. In order to prevent these additional switchings, two extra conditions are required as follows :

$$
\begin{aligned}
& x(t)>0 \quad t \in\left(0, \pi / \omega^{-}\right) . \\
& x(t)<0 \quad t \in\left(\pi / \omega, 2 \pi / \omega^{-}\right) .
\end{aligned}
$$

If conditions (4)-(6) are satisfied at $\omega=\omega_{f}$ where $\omega_{f}$ is the frequency of $f(t)$, then FO occurs. If $\omega=\omega_{f} / \nu, \nu$ odd integer, then $\mathrm{SO}$ of order $\nu$ is said to have taken place.

In the RFS of Fig. 1, if SO of order $\nu$ occurs, then the relay switches once every half period, corresponding to $\nu T_{f} / 2$. Hence, within any half period, the state response of $G(s)$, of the form in (1), should be written separately - one segment, $z_{1}(t)$, corresponding to a response to a negative value of the relay output and the other segment, $z_{2}(t)$, corresponding to the positive relay output. This is because, $t=0$ corresponds to a positive relay switch and therefore, for $G(s)$ with a 
delay $L$, the response between $t=0$ and $t=L$ is due to a negative relay output while the response between $t=L$ and $t=\nu T_{f} / 2$ is due to the positive relay output. Thus, when periodic switching of frequency $\omega_{f} / \nu$ takes place, the state response of the plant is given by

$$
\begin{aligned}
& z_{1}(t)=e^{A t} z(0)+\left(I-e^{A t}\right) A^{-1} B h, t \in[0, L] \\
& z_{2}(t)=e^{A t} z(0)+\left(2 e^{A(t-L)}-e^{A t}-I\right) A^{-1} B h, t \in\left[L, \nu \frac{T_{f}}{2}\right]
\end{aligned}
$$

where $z(0)$ is the value of the state vector at the positive switching edge of the relay output. $z(0)$ as derived in [9] is given by :

$$
\begin{aligned}
& z(0)=-z\left(\frac{\nu T_{f}}{2}\right) \\
& =-\left(I+e^{A \nu \frac{T_{f}}{2}}\right)^{-1}\left(2 e^{A\left(\nu \frac{T_{f}}{2}-L\right)}-e^{A \nu \frac{T_{f}}{2}}-I\right) A^{-1} B h .
\end{aligned}
$$

Since in the RFS of Fig. 1, $y(t)=c(t)+f(t)$, we have

$$
\begin{aligned}
y_{1}(t) & =C e^{A t} z(0)+C\left(I-e^{A t}\right) A^{-1} B h \\
& +R \sin \left(\omega_{f} t+\theta\right), \quad t \in[0, L] . \\
y_{2}(t) & =C e^{A t} z(0)+C\left(2 e^{A(t-L)}-e^{A t}-I\right) A^{-1} B h \\
& +R \sin \left(\omega_{f} t+\theta\right), \quad t \in\left[L, \nu \frac{T_{f}}{2}\right] .
\end{aligned}
$$

The necessary and sufficient conditions for SO are presented in Proposition 1.

Proposition 1: The RFS in Fig. 1 has a symmetric unimodal periodic solution with a half period of $\nu T_{f} / 2$ where $\nu \geq 3$ and odd if and only if the following conditions are satisfied

$$
\begin{aligned}
& y_{1}(0)=0, \quad y_{2}\left(\nu \frac{T_{f}}{2}\right)=0 \\
& y_{1}(t)<0, \quad t \in(0, L] \\
& y_{2}(t)<0, \quad t \in\left[L, \nu \frac{T_{f}}{2}\right)
\end{aligned}
$$

where $0<L<\nu T f / 2$ and $z(0)$ is as defined in (7).

\section{Analysis of (10)-(12)}

From (10), by substituting $t=\frac{\nu T_{f}}{2}$ in (9), we get

$$
\begin{aligned}
R \sin \theta & =C z\left(\frac{\nu T_{f}}{2}\right)=-C z(0) \\
\theta & =\pi+\operatorname{sign}(C z(0)) \sin ^{-1} \frac{|C z(0)|}{R}
\end{aligned}
$$

and we require the minimum $R$ to satisfy

$$
R \geq|C z(0)| .
$$

Substituting (14) into (11),

$$
\begin{aligned}
& C e^{A t} z(0)+C\left(I-e^{A t}\right) A^{-1} B h \\
& \quad+R \sin \left(\omega_{f} t+\pi+\operatorname{sign}(C z(0)) \sin ^{-1} \frac{|C z(0)|}{R}\right)<0 \\
& \Rightarrow \sin \left(\omega_{f} t\right) \operatorname{sign}(C z(0)) \sqrt{R^{2}-(C z(0))^{2}}>C e^{A t} z(0) \\
& \quad+C\left(I-e^{A t}\right) A^{-1} B h-\cos \left(\omega_{f} t\right) C z(0) .
\end{aligned}
$$

Note that at $t=m \frac{T_{f}}{2}$ for $m \in \mathbb{N}^{+}$and $m<\nu$, both sides of (16) are identically zero. Thus, (16) is only valid for all $t \in(0, L)$, excluding $t=m \frac{T_{f}}{2}$ for $m \in \mathbb{N}^{+}$and $m<\nu$.

From (16), it follows that the condition on $R$ satisfies

$$
\begin{aligned}
& \sqrt{R^{2}-(C z(0))^{2}}>C\left(e^{A t} z(0)+\left(I-e^{A t}\right) A^{-1} B h\right. \\
& \left.\quad-\cos \left(\omega_{f} t\right) z(0)\right) /\left|\sin \left(\omega_{f} t\right) \operatorname{sign}(C z(0))\right|
\end{aligned}
$$

where $t \in(0, L)$, excluding $t=m \frac{T_{f}}{2}$ for $m \in \mathbb{N}^{+}$and $m<\nu$.

Similarly, substituting (14) into (12), we obtain

$$
\begin{gathered}
\sqrt{R^{2}-(C z(0))^{2}}>C\left(e^{A t} z(0)+\left(2 e^{A(t-L)}-e^{A t}-I\right)\right. \\
\left.A^{-1} B h-\cos \left(\omega_{f} t\right) z(0)\right) /\left|\sin \left(\omega_{f} t\right) \operatorname{sign}(C z(0))\right|
\end{gathered}
$$

where $t \in\left(L, \frac{\nu T_{f}}{2}\right)$, excluding $t=m \frac{T_{f}}{2}$.

Given $\nu$ and $\omega_{f}$, the set of $R$ that satisfies (15), (17) and (18) can now be determined. Denote this set as $R \geq$ $R_{\nu, \min }\left(\omega_{f}\right)$ where $R_{\nu, \min }($.$) is the minimum R$ that satisfies (15), (17) and (18) for $t$ defined in $t \in\left(0, \frac{\nu T_{f}}{2}\right)$, excluding $t=m T_{f} / 2$. We now examine conditions (11) and (12) at $t=m T_{f} / 2$ where $m \in \mathbb{N}^{+}$and $m<\nu$.

Using (13), for $m \in \mathbb{N}^{+}$,

$$
R \sin (m \pi+\theta)=(-1)^{m+1} C z(0) .
$$

Substituting $t=m T_{f} / 2$ and (19) into (11), we have

$$
\begin{aligned}
C e^{A \frac{m T_{f}}{2}} z(0)+ & C\left(I-e^{A \frac{m T_{f}}{2}}\right) A^{-1} B h \\
& +(-1)^{m+1} C z(0)<0 .
\end{aligned}
$$

Substituting $t=m T_{f} / 2$ and (19) into (12),

$$
\begin{array}{r}
C e^{A \frac{m T_{f}}{2}} z(0)+C\left(2 e^{A\left(\frac{m T_{f}}{2}-L\right)}-e^{A \frac{m T_{f}}{2}}-I\right) \\
A^{-1} B h+(-1)^{m+1} C z(0)<0 .
\end{array}
$$

Observe that (20) and (21) are independent of $R$ but dependent on $\nu T_{f}$. On the other hand, $R$ is constrained by (15), (17) and (18) which is also dependent on $\nu T_{f}$. Let $\omega_{1}<\omega_{f}<\omega_{2}$ be the range of frequencies which satisfies (20) and (21) while the minimum value of $R$ which satisfies (15), (17) and (18) is denoted by $R_{\nu, \min }\left(\omega_{f}\right)$. Then the above analysis implies that for a given $f(t)$ with an amplitude $R$ and frequency $\omega_{f 0}$, if

$$
R_{1, \min }\left(\omega_{f 0}\right)>R \geq R_{\nu, \min }\left(\omega_{f 0}\right)
$$

where $\omega_{1} \leq \omega_{f 0} \leq \omega_{2}$, then SO of order $\nu$ should be observable in the RFS. $R_{1, \min }$ denotes the minimum $R$ for SO of order $\nu=1$ to occur. This is equivalent to forced oscillation taking place at the frequency of $f(t)$. The following example illustrates the analysis for a first order delayed (FOPDT) plant.

Example 1: Consider a FOPDT plant with $A=B=$ $C=L=1$ and zero initial conditions. Values of $R_{\nu, \min }$ are plotted against $T_{f} / 2$ in Fig. 3 where $\omega_{f}=2 \pi / T_{f}$ corresponds to the frequency of $f(t)$. In this example, the values of $R_{\nu, \min }$ are found to be equal to $|C z(0)|$ as in 


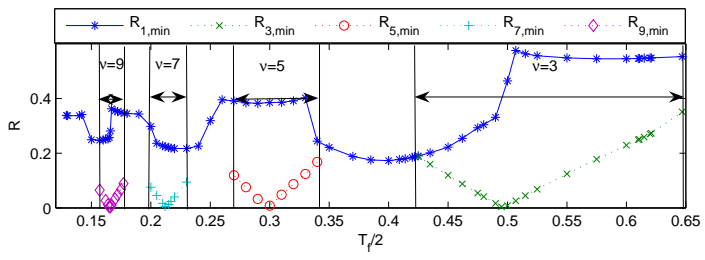

Fig. 3. Dependence of SO on $R$ and $T_{f} / 2$.

(15) and they also satisfy (17) and (18). The $R_{1, \min }$ values for forced oscillation are derived from [7]. If $T_{f} / 2$ of the external forcing signal falls within the bounds indicated in Fig. 3 for each $\nu$ and $R_{1, \min }>R \geq R_{\nu, \min }$, the conditions for the existence of SO of order $\nu$ are satisfied and SO will be observed. For example, SO with $\nu=3$ will occur for the range $0.418<T_{f} / 2<0.643$ if $R_{1, \min }>R \geq R_{3, \text { min }}$. Fig. 4 shows $\mathrm{SO}$ of $\nu=3$ when $T_{f} / 2=0.6$ and $R_{1, \min }=$ $0.545>R=0.2289>R_{3, \min }=0.2288$.

Observe also that for a particular $R$ in Fig. 3, SO of different $\nu$ s should theoretically be obtained by varying the frequency of $f(t)$. Fig. 5 shows a plot of the relay switching time intervals against $t_{i}$ for a fixed $R=0.145$ where $t_{i}$ denotes the relay switching time. The frequency of $f(t)$ was varied in the simulation to obtain the different orders of SO. As can be seen, SO of orders, $\nu=3,5,7,9$ were observed at different frequencies while $R$ was fixed at 0.145 .

In the next section, the existence of SO with different orders for different plants is analysed and simulation results are presented.

\section{Analysis and Simulation Results}

The inequalities in (20) and (21) will now be analysed to extract valuable information on the range of frequencies, $\omega_{f}$ of $f(t)$, where various orders of SO can occur. The analysis is presented for different classes of plants.

\section{A. SO analysis for first order non-delayed plants}

For this class of plants, self oscillations do not occur in the RFS of Fig. 1. However, FO can still take place for a sufficiently large $R$. Using the analysis in Section III, it can be shown that SO cannot occur (despite the existence of FO) due to a violation of (21) at $t=k T_{f}$ where $k \in \mathbb{N}^{+}$. This is shown as follows :

$$
y_{2}\left(k T_{f}\right)=C\left(z_{2}\left(k T_{f}\right)+(-1)^{2 k+1} z(0)\right)>0 \text { for all } \nu .
$$

Since (22) violates (21) for all $\nu$, no SO can take place for this class of plants.

\section{B. SO analysis for first order plants with delay}

For FOPDT plants, self oscillation is possible due to the effect of the delay. For a sufficiently large $R$, they also exhibit

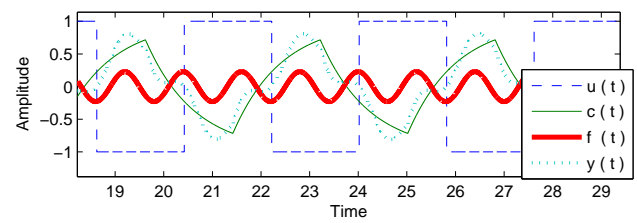

Fig. 4. Example where the desired $\mathrm{SO}$ with $\nu=3$ is obtained.

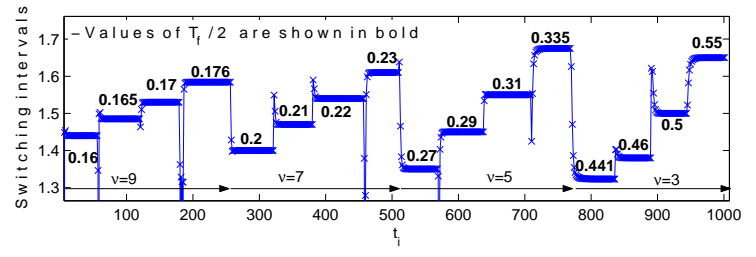

Fig. 5. Different $\nu$ s obtained with a fixed $R$ and varying $T_{f} / 2$.

FO. For FOPDT plants, it can be shown that SO of order $\nu \geq 2 n+3$ cannot occur for frequencies satisfying

$$
n T_{f} / 2<L<(n+1) T_{f} / 2
$$

where $n \in \mathbb{N}$.

$$
\begin{aligned}
& \text { At } t=(n+1) T_{f}, \text { substituting } \nu \geq 2 n+3 \text { into (9), } \\
& y_{2}\left((n+1) T_{f}\right) \\
& =C\left[z\left((n+1) T_{f}\right)+(-1)^{2 n+3} z(0)\right] \\
& =2 C\left(I+e^{A \nu \frac{T_{f}}{2}}\right)^{-1}\left[e^{A\left(\nu \frac{T_{f}}{2}-L\right)}+e^{A\left((n+1) T_{f}-L\right)}\right. \\
& \\
& \left.-e^{A \nu \frac{T_{f}}{2}}-I\right] A^{-1} B h>0
\end{aligned}
$$

which clearly violates (21). Hence SO of order, $\nu \geq 2 n+$ 3 cannot exist. An important result to note is that for low external frequencies satisfying $n=0$ in (23), only FO is possible and no $\mathrm{SO}$ of any order can occur for this class of plants.

Example 2: Consider a FOPDT plant, where $A=-1 / 3$, $B=1, C=1 / 3$ and $L=2$. Using (20) and (21), the bounds of $T_{f} / 2$ for each $\nu$ can be calculated. These are plotted in Fig. 6 along with the possible $\nu$ s obtained from simulations. Note that for a given $L, T_{f} / 2$ can also be parameterized in terms of $n$ according to (23). The figure shows that $\mathrm{SO}$ of order $\nu \geq 2 n+3$ cannot occur. For example, for $n=2$, only $\nu=5$ is possible while $\nu \geq 7$ is not. From Fig. 6, it can also be observed that $0<L<\nu \frac{T_{f}}{2}$ and at each $T_{f} / 2$, only a single order of SO exists. This is equivalent to the result where for each and every order of SO, their frequencies do not overlap.

This can be shown as follows. For $n \geq 1$, at $t=T_{f} / 2$ and $t=n T_{f}$, SO exists if

$$
\begin{aligned}
y_{1}\left(\frac{T_{f}}{2}\right) & =C\left(I+e^{A \nu \frac{T_{f}}{2}}\right)^{-1}\left[-e^{A\left((\nu+1) \frac{T_{f}}{2}-L\right)}-e^{A\left(\nu \frac{T_{f}}{2}-L\right)}\right. \\
& \left.+e^{A \nu \frac{T_{f}}{2}}+I\right] A^{-1} B h<0 . \\
y_{2}\left(n T_{f}\right) & =C\left(I+e^{A \nu \frac{T_{f}}{2}}\right)^{-1}\left[e^{A\left(n T_{f}-L\right)}+e^{A\left(\nu \frac{T_{f}}{2}-L\right)}\right. \\
& \left.-e^{A \nu \frac{T_{f}}{2}}-I\right] A^{-1} B h<0 .
\end{aligned}
$$

(25) and (26) leads to

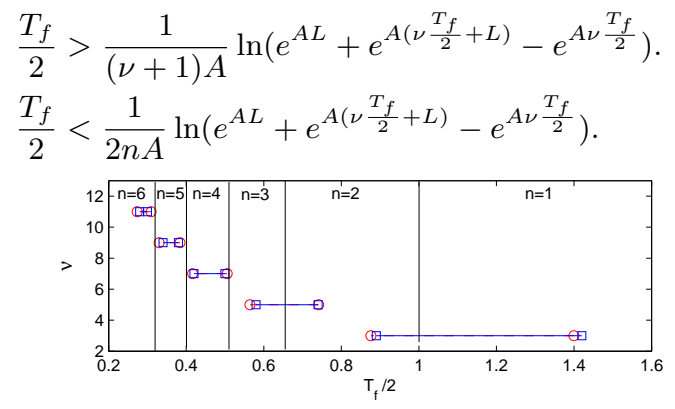

Fig. 6. Plot of the bounds for example 2, 'o': Calculated, ' $\square$ ': Simulated. 


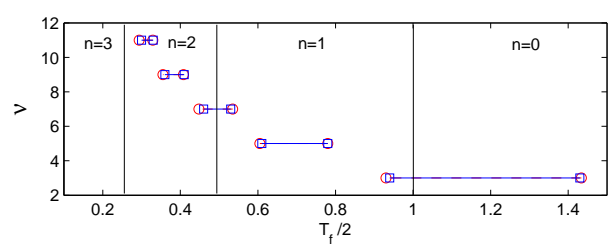

Fig. 7. Plot of the bounds for example 3, 'o': Calculated, ' $\square$ ':Simulated.

Let $T_{f} / 2=h_{1}$ satisfy (27). If $2 n>\nu+1$, then $T_{f} / 2=$ $h_{1}$ will never satisfy (28). For FOPDT plants, $\nu<2 n+3$ thus $2 n>\nu-3$. So (27) and (28) can be satisfied for a common range of frequencies where (25) and (26) provide the corresponding lower and upper bounds of frequencies where SO of a certain $\nu$ can exist. In example 1 , at $n=1$ and $\nu=3, T_{f} / 2>0.418$ satisfies (25) and $T_{f} / 2<0.643$ satisfies (26). Hence, $\nu=3$ occurs for $0.418<T_{f} / 2<$ 0.643 .

Next we will show that the range of frequencies, $\omega_{f}$ for adjacent orders, $\nu=2 j-1$ and $\nu=2 j+1$ will not overlap where $j \in \mathbb{N}^{+}$and $j>1$. Recall that for FOPDT plants, only SO with $\nu<2 n+3$ will exist. Thus, if $n=j$, only $\nu=2 j-1$ and $2 j+1$ can exist.

Rearranging the terms in (27) and (28),

$$
\begin{aligned}
& \text { (27): } \frac{T_{f}}{2}>\frac{1}{\nu A}\left[\ln \left(e^{A L}-e^{(\nu+1) A \frac{T_{f}}{2}}\right)-\ln \left(I-e^{A L}\right)\right] . \\
& \text { (28): } \frac{T_{f}}{2}<\frac{1}{\nu A}\left[\ln \left(e^{A L}-e^{n A T_{f}}\right)-\ln \left(I-e^{A L}\right)\right] .
\end{aligned}
$$

Substituting $\nu=2 j-1$ into (29) will give the lower bound on the range of $\omega_{f}$ where SO of order $\nu=2 j-1$ exist.

$$
\text { (29) : } \frac{T_{f}}{2}>\frac{1}{(2 j-1) A}\left[\ln \left(e^{A L}-e^{j A T_{f}}\right)-\ln \left(I-e^{A L}\right)\right] \text {. }
$$

Substituting $\nu=2 j+1$ and $n=j$ in (30) will give the upper bound on the range of $\omega_{f}$ where SO of order $\nu=2 j+1$ exist.

$$
\text { (30) : } \frac{T_{f}}{2}<\frac{1}{(2 j+1) A}\left[\ln \left(e^{A L}-e^{n A T_{f}}\right)-\ln \left(I-e^{A L}\right)\right] \text {. }
$$

If $T_{f} / 2=h_{2}$ satisfies (31), it will never satisfy (32). So the lower bound on the $\omega_{f}$ for $\nu=2 j-1$ will not overlap with the upper bound for $\nu=2 j+1$. So the range of frequencies for $\nu=2 j-1$ and $\nu=2 j+1$ where $j \in \mathbb{N}^{+}$, will not overlap and for each $T_{f} / 2$, only a single order of SO can exist. This is confirmed in Fig. 6.

\section{SO analysis for higher order plants}

FO behaviours of the higher order plants generally follow that of the first order plants. However, the self oscillation behaviours vary and the SO behaviours differ greatly from the first order plants. For the higher order plants with delay, (21) can be satisfied for some $\omega_{f}$ by substituting $n=0$,

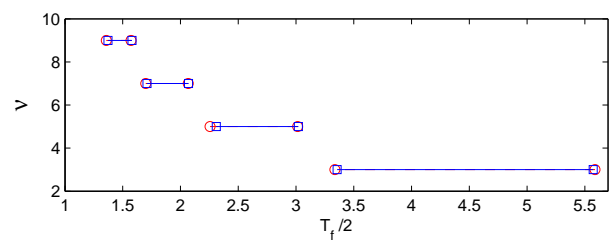

Fig. 8. Plot of the bounds for example 4, 'o': Calculated, ' $\square$ ':Simulated.

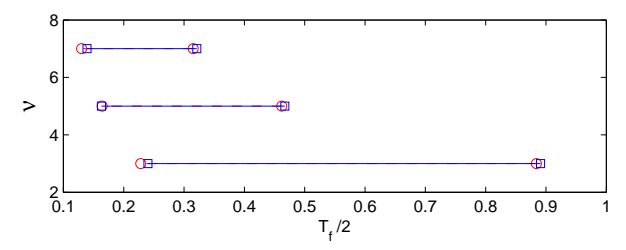

Fig. 9. Plot of bounds for example 5, 'o': Calculated, ' $\square$ ':Simulated.

$\nu=3$ into (24). Therefore, $\nu \geq 2 n+3$ can exist unlike SO conditions for FOPDT plants.

Example 3: Consider a third order delayed plant, $G(s)=$ $\frac{e^{-s}}{(s+1)^{3}}$. The range of $T_{f} / 2$ for which each $\nu$ exists is plotted in Fig. 7. It can be observed that for some $T_{f} / 2$ corresponding to $n \geq 0$, SO of order $\nu \geq 2 n+3$ cannot be excluded. For example, for $n=1$, SO of orders $\nu=3,5,7$ do exist.

Recall from Section IV-A, that for first order non-delayed plants, no SOs of any order can occur. This is in contrast to higher non-delayed plants as shown in the following example.

Example 4: Consider a fourth order non-delayed plant, $G(s)=\frac{-s+0.2}{s^{4}+2 s^{3}+1.31 s^{2}+0.34 s+0.03}$. Fig. 8 shows the ranges of frequencies for $\nu=3,5,7,9$ where a single order of $\nu$ was observed for a given $T_{f} / 2$. Fig. 6,7 and 8 also show that the predicted bounds are a good indication of the actual bounds on the range of $T_{f} / 2$ where $\mathrm{SO}$ of a certain $\nu$ can occur. The bounds do not overlap and thus only a single order of SO exist for a given $T_{f} / 2$.

The following example shows that for some plants, several $\nu$ s can occur for a given $T_{f} / 2$. The conditions under which they happen depend on the $R$ value of $f(t)$ as well as the initial states of $G(s)$ and $\theta$.

Example 5: Consider a second order non-delayed plant, $G(s)=\frac{-s+0.2}{s^{2}+6 s+7}$ with zero initial state vector. Fig. 9 shows the plot of the calculated bounds against the simulated bounds for $\nu=3,5,7$. These bounds overlap for $\nu=3,5,7$ indicating that multiple orders of SO are possible at these frequencies and the actual order that occurs depend on the magnitude of $R$.

Fig. 10 is a plot of the relay switching time intervals when the RFS was driven by a $f(t)$ with varying amplitude $R$ and fixed frequency corresponding to $\frac{T_{f}}{2}=0.12$. The amplitude $R$ of $f(t)$ was varied in the simulation to obtain the different orders of SO. $R$ was varied as shown in Table I while $T_{f}$ was fixed at 0.12 . Initially, $R=0.12$ and FO with $\nu=1$ was observed. After a period of time, $R$ was set to $R=0.0871$ and $\nu=9$ was observed even though the frequency of $f(t)$ remained unchanged. By changing $R$ further, SO with $\nu=$ $11,13,15,17$ was observed to be possible. Next, the effect of the initial conditions on the order of SO observed will be shown.

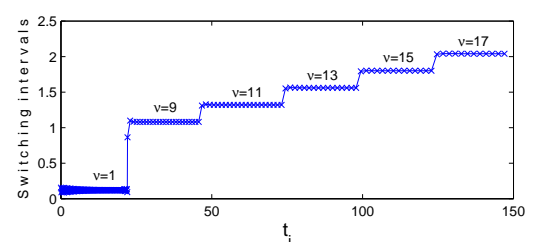

Fig. 10. Multiple $\nu$ s of SO observed for example 5 with varying $R$. 


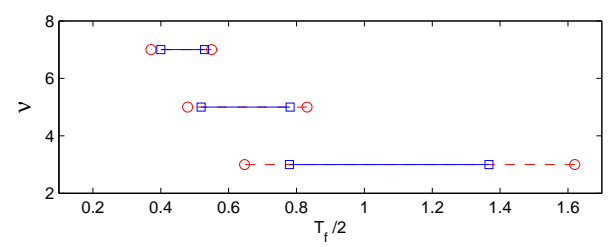

Fig. 11. Effect of only varying $R$ for example 6, 'o': Calculated bounds, ' $\square$ ':Simulated bounds.

TABLE I

TABLE OF $R$ AND $R_{\nu, \text { min }}$ FOR EXAMPLE 5 .

\begin{tabular}{|c|c|c|c|c|c|c|}
\hline$\nu$ & 1 & 9 & 11 & 13 & 15 & 17 \\
\hline$R_{\nu, \min }$ & 0.11 & 0.087 & 0.057 & 0.033 & 0.015 & 0.0015 \\
\hline$R$ & 0.12 & 0.0871 & 0.0571 & 0.0331 & 0.0151 & 0.0016 \\
\hline
\end{tabular}

Example 6: Consider $G(s)=\frac{s+0.2}{s^{4}+0.03 s^{3}+0.34 s^{2}+1.31 s+2}$. The bounds of $T_{f} / 2$ for each $\nu$ are shown in Fig. 11. It is clear that the bounds overlap but it appears that the predicted bounds are not accurate when compared to the simulated bounds. Fig. 11 was obtained under zero initial conditions of the state vector. The overlapping bounds indicate that different $\nu \mathrm{s}$ are possible under the right conditions.

At $T_{f} / 2=0.699$, when the initial state vector was changed to $z(0)=[-0.3955 ;-0.4220 ; 0.09896 ; 0.1838]$ and $f(t)$ was set with $R=0.1358>R_{3, \min }=0.1357$ and $\theta=4.6783 \mathrm{rad}$, SO of order $\nu=3$ occurred at the first switch. When $\theta$ was reset to $\theta=0, \nu=5$ occurred after some initial transients. The oscillations are shown in Fig. 12. This result confirms the consequences of the overlapping bounds in Fig. 12 which imply that $\nu=3$ and $\nu=5$ are possible under the right conditions.

By further varying the initial state vector and $\theta$, it was possible to obtain simulated bounds of $T_{f} / 2$ which are closer to that predicted from our analysis. This is shown in Fig. 13 for the same $G(s)$ in this example. The two bounds are now almost identical. The effect of varying the initial state vector is best illustrated in Fig. 14 which shows a plot of the state $z_{3}$ against $z_{4} . R$ is fixed at 0.26931 and the initial state vector, $z_{0}$ is varied from $[0 ; 0 ; 0 ; 0]$ to $[1 ; 1 ; 0 ; 0.5]$. It can be seen that the trajectories of $z(t)$ tend towards two different limit cycles of orders, $\nu=3$ and $\nu=5$.

\section{CONCLUSION}

In this paper, the frequency ranges where $\mathrm{SO}$ of a certain order $\nu$ can exist, have been predicted. It was seen in the

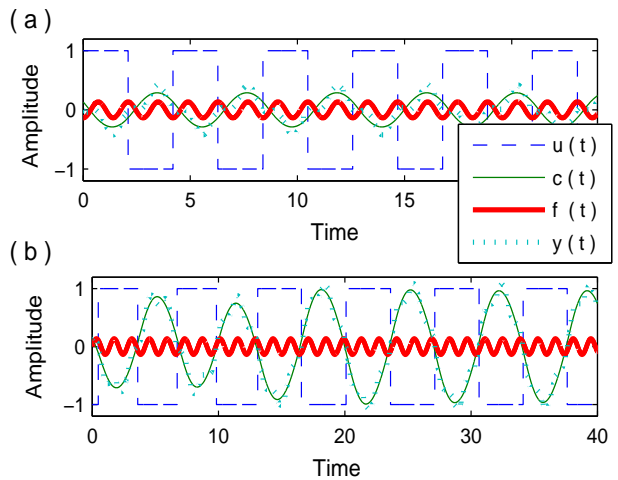

Fig. 12. (a) SO of $\nu=3$ obtained with $\theta=4.6783$. (b) SO of $\nu=5$ obtained with $\theta=0$.

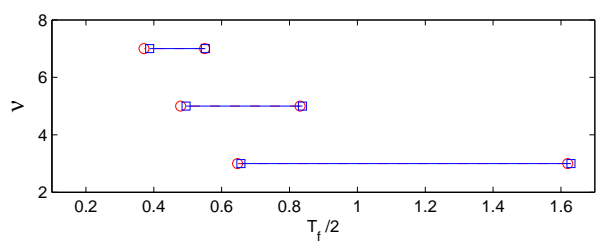

Fig. 13. Effect of varying $R, z_{0}$ and $\theta$ for example 6, 'o': Calculated bounds, ' $\square$ ': Simulated bounds.

analysis that SO will not exist in first order non-delayed plants. Single order of SO for a fixed $\omega_{f}$ will occur for first order plants with delay and SO of order, $\nu \geq 2 n+3$ will not exist. Single or multiple orders of SO for a fixed $\omega_{f}$ are possible for higher order plants. It was found that the existence of SO in RFS is dependent on several parameters, the amplitude $R$ of the external forcing signal, the initial state vector $z_{0}$, the initial phase offset $\theta$ of the external signal and the external forcing frequency, $\omega_{f}$. A variation in any of these parameters can lead to a different SO behaviour. Due to the complex behaviours involved, the prediction on the ranges of frequencies where SO can exist is highly desirable. It would be extremely tedious and inconvenient if the possibility of SO existence has to be checked by searching through all initial conditions. The predicted ranges of frequencies for the existence of SO of a certain order $\nu$ have been verified to be good for first order delayed plants and higher order delayed and non-delayed plants.

\section{REFERENCES}

[1] Mario Di Bernado, Karl Johansson, "Self-Oscillations and Sliding in Relay Feedback Systems: Symmetry and Bifurcations", Intl Journal of Bifurcation and Chaos, Vol. 11, No. 4, 2001

[2] Tsypkin Y Z, Relay Control Systems, Cambridge University Press, 1984.

[3] Chong Lin, Qing-Guo Wang, Tong Heng Lee and James Lam, "Local Stability of Limit Cycles for Time-Delay Relay-Feedback Systems", IEEE Transactions on Circuits and Systems Part I, vol. 49, no. 12, December 2002

[4] Karl Henrik Johansson, Anders Rantzer, Karl Johan Astrom, "Fast Switches in relay feedback systems", Automatica, 35, pp 539-552, 1999

[5] Leonid M. Fridman, "Singular Perturbed Analysis of Chattering in Relay Control Systems", IEEE Trans on Automatic Control, 2002

[6] J.M. Goncalves, A. Megretshi and M.A. Dahleh, "Global Stability of relay feedback systems", Technical Report Preprint LIDS-P-2458, Dept. of EECS, MIT, Cambridge, MA, 1999

[7] Lim L H, Loh A P, Fu J, "Estimation of Minimum Conditions for Forced Oscillation in Relay Feedback Systems", ICCA, pp 1262-1267, 2005

[8] Atherton D P, Nonlinear Control Engineering, Describing Analysis and Design, Van Nostrand Reinhold, 1982.

[9] K.J.Åström, "Oscillations in systems with relay feedback", in Adaptive Control, Filtering and Signal Processing, K.J.Astrom, G.C.Goodwin and P.R.Kumar Eds, vol. 74, IMA Volumes in Mathematics and its Applications, pp 1-25, Springer-Verlag, 1995

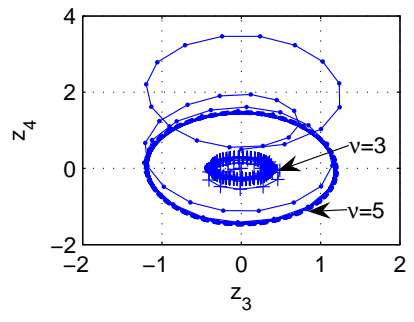

Fig. 14. Effect of the initial condition for example 6 . 